\title{
Die „Hamburger Rechtsstudien“ erscheinen in zwangloser Folge und sind einzeln in jeder Buchhandlung käuflich
}

Bisher sind erschienen:

Heft 1: Der Begriff des Versicherungsfalles in der Seeversicherung. Von Dr. F. Alexander Bene. GroB-Oktav. 75 Seiten. 1928.

RM 405

Heft 2: Die Bedeutung des Interesses für die Veräußerung der versicherten Sache. Von Dr. Hermann Heinrich Elkan. Groß-Oktav. 58 Seiten. 1928. RM 3.60

Heft 3: Aktiensonderdepot und Legitimationsübertragung. Von Dr. Günther Frohner. GroB-Oktav. 121 Seiten. 1929 RM 6.30

Heft 4: Die Gewinnversicherung. Von Dr. Helmut Winkler. GroB-Oktav. 31 Seiten. 1930.

RM 1.80

Heft 5: Der Konnossement-Teilschein. Von Dr. Heinz Behlert. GroB-Oktav. 79 Seiten. 1930.

RM 4.50

Heft 6: Die Order-Police. Von Dr. Alexander N. Tsirintanis. Groß-Oktav. 85 Seiten. 1930.

RM 5.40

Heft 7: Reine Konnossemente gegen Revers. Von Dr. Robert Lion. GroB-Oktav. 78 Seiten. 1930.

RM 4.50

Heft 8: Versicherung für Rechnung wen es angeht. Von Dr. Helmuth Embden. Groß-Oktav. 39 Seiten. 1930.

RM 2.70

Heft 9: Die guten Sitten in der arbeitsrecttlichen Rechtsprechung nach dem Kriege. Von Dr. Fritz Oettinger. GroB-Oktav. 84 Seiten. 1931. RM 4.50

Heft 10: Wandlung und Minderung bei einer Mehrheit von Käufern oder Verkäufern. Von Dr. Hans Wogatzky. Groß-Oktav. 115 Seiten. 1931. RM 6.-

Heft 11: Das Versicherungs-Zertifikat. Von Dr. Rudolf Nothmann. GroB-Oktav. 96 Seiten. 1932. RM 5.-

Heft 12: Die Versicherung der Havariegrosse-Scäden. Von Dr. Hans Cramer. Groß-Oktav. 56 Seiten. 1932. RM 3.-

Heft 13: Die Staatshaftung für den Hamburger Hafenlotsen. Von Dr. Erwin Mumssen. GroB-Oktav. 110 Seiten. 1932. RM 5.-

Heft 14: Gleichberechtigung der Geschlechter im künftigen Elternrecht. Von Dr. Charlotte Cohn. Groß-Oktav. XI u. 56 Seiten. $1932 . \quad$ RM 3.50

Heft 15: Die Speditionsversicherung in den Allgemeinen Deutschen Spediteurbedingungen. Von Dr. Willi Schiering. GroB-Oktav.74 Seiten. 1932.

RM 4.-

Heft 16: Quellenkritische Studien zur Bessergebotsklausel (in diem addictio) im römischen Kaufrecht. Von Dr. jur. Harald Sieg. Groß-Oktav. 43 Seiten. 1933.

RM 3.-

Demnächst erscheint:

Heft 18: Acatholicus. Eine Untersuchung über die Stellung der Ungetauften und der Apostaten, Häretiker und Schismatiker sowie der sonstigen exkommunizierten Christen im geltenden kanonischen Recht. Von Dr. Wulter Bohm. GroB-Oktav. 59 Seiten. 1933. 



\section{Kostfrachtgeschäft und}

\section{laufende Versicherung}

von

Dr. iur. Detlev Himer

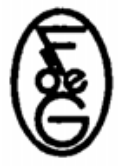

Hamburg

Friederichsen, de Gruyter \& Co. m. b. H.

1933 
Diese Arbeit ist als Doktordissertation von der Rechtsund Staatswissenschaftlichen Fakultät

der Hamburgischen Universität angenommen worden.

Gedrudkt bei C. Trute, Quakenbrüdk i. H. 
Meinen Eltern! 
